\title{
Single Photon HgCdTe Avalanche Photodiode and Integrated Detector Cooler Assemblies for Space Lidar Applications
}

\author{
Xiaoli Sun*a James Abshire, Michael Krainak, Wei Lu ${ }^{\mathrm{a}}$ \\ Jeff Beck ${ }^{\mathrm{b}}$, William Sullivan III ${ }^{\mathrm{b}}$, Pradip Mitra ${ }^{\mathrm{b}}$, Dick Rawlings ${ }^{\mathrm{b}}$ \\ Renny Fields ${ }^{c}$, David Hinkley ${ }^{c}$, and Bradley Hirasuna ${ }^{c}$ \\ aNASA Goddard Space Flight Center, Greenbelt MD USA 20771; \\ bLeonardo DRS Electro-Optical Infrared Systems, Dallas, TX USA 75374; \\ ${ }^{\mathrm{c}}$ The Aerospace Corporation, El Segundo, CA USA 90245
}

\begin{abstract}
A linear mode photon counting $\mathrm{HgCdTe}$ avalanche photodiode (APD) focal plane array (FPA) detector was developed for space lidar applications. An integrated detector cooler assembly (IDCA) was manufactured using a miniature Stirling cooler. The HgCdTe APD demonstrated a greater than $60 \%$ photon detection efficiency from 0.9 to $4.3 \mu$ m wavelength and a dark count rate less than $250,000 / \mathrm{s}$. The IDCA cooled the FPA to $110 \mathrm{~K}$ from ambient room temperature at a total electrical power of $7 \mathrm{~W}$. The IDCA has passed environmental tests, including vibration, thermal cycling and thermal vacuum tests.
\end{abstract}

Keywords: photon counting, infrared detectors, cryo-coolers, lidar

\section{INTRODUCTION}

Evaluations have shown that $\mathrm{HgCdTe}$ avalanche photodiode (APD) focal plane arrays (FPA) have linear mode photon counting with $>60 \%$ photon counting efficiency up to mid-infrared wavelengths. ${ }^{1}$ They are near ideal detectors for the next generation space lidar for surface elevation, spectral absorption, atmosphere gas concentration, and backscatter profile measurements. They have enabled airborne and space-borne lidar to operate in near- to mid-infrared laser wavelengths to simultaneously map the surface topography and distribution of various elements such as water ice on the moon, asteroids, and comet cores.

NASA's Earth Science Technology Office (ESTO) previously supported Goddard Space Flight Center (GSFC) to collaborate with Leonardo DRS Electro-Optical Infrared Systems to mature the fabrication of the HgCdTe APD arrays and to develop a cryo-cooler and packaging approach to use as detectors in multi-beam laser altimeters and for IPDA lidar for $\mathrm{CO}_{2}$ and $\mathrm{CH}_{4}$. As part of this work DRS produced a batch of $4 \mathrm{x} 4$ pixel $\mathrm{HgCdTe}$ APD arrays that have been successfully used in GSFC's airborne $\mathrm{CO}_{2}$ and $\mathrm{CH}_{4}$ lidar where they showed nearly quantum limited receiver performance. ${ }^{2}$ DRS also produced a set of $2 \times 8$ pixel prototype devices with even lower dark current and higher APD gain that was sufficient for applications requiring linear mode photon counting (LMPC). ${ }^{3}$ Recently DRS successfully integrated a 2x8 pixel $\mathrm{HgCdTe}$ APD FPA with a microlens array into a mini-Stirling cooler into an integrated detector cooler assembly (IDCA). ${ }^{4}$ That detector has been characterized and the results are summarized here.

The 2x8 pixel HgCdTe APD FPA's have $64 \times 64 \mu \mathrm{m}^{2}$ pixel size and $64 \mu \mathrm{m}$ pitch. Test results showed they had a $60 \%$ photon counting efficiency at $1.55 \mu \mathrm{m}$ wavelength and $<250 \mathrm{kHz}$ noise count rate in LMPC mode operation. A major portion of the dark counts was found to come from the ambient thermal emissions through the gaps in the cold shield in the initial test setup. The actual dark current of the $\mathrm{HgCdTe}$ APD was measured to be $<50 \mathrm{~K}$ electrons/s per pixel. The analog outputs of the detectors had an impulse response of $8 \mathrm{~ns}$ and the amplitude was proportional to the incident number of photons per pulse. The analog output pulse width was limited by the preamplifier bandwidth. There was also a set of built-in comparators, one for each pixel, that could turn the analog signal for each detected photon into a digital pulse. The devices could be operated in either analog or digital output modes, or both.

*xiaoli.sun-1@nasa.gov; phone 1 (301) 614-6732; fax 1 (301) 614-6744 
Two IDCA's were fabricated and characterized. Environmental tests were performed to these IDCA's, including a vibration test, a thermal cycle test, a thermal shock test, and a thermal vacuum tests. Radiation damage tests were performed as well, which demonstrate these devices can be used in the space radiation environment in a typical Earth orbiting radiation environment. ${ }^{5}$ The $\mathrm{HgCdTe}$ APD array, the cryo-cooler, and the IDCA will be described in detail in this paper. The test results of the LMPC HgCdTe APD FPAs and the IDCA at both DRS and GSFC will be reported.

\section{THE HGCDTE APD LMPC FPA}

\subsection{HgCdTe APD arrays}

The DRS LMPC HgCdTe APD arrays are similar to their high-density vertically-integrated-photodiode (HDVIP ${ }^{\circledR}$ ) $\mathrm{HgCdTe}$ APD arrays. Each element is a p-around-n cylindrical photodiode formed around small via in the HgCdTe film on silicon substrate. ${ }^{6-9}$ Each pixel consists of four photodiodes connected in parallel. The same pixel structure can be replicated into an array of pixels of desired format. $\mathrm{HgCdTe}$ APDs have a wide spectral response from the visible to the cutoff wavelength where the cut-off wavelength is determined by the mole fraction $\mathrm{x}$ of $\mathrm{CdTe}$ in $\mathrm{Hg}_{1-\mathrm{x}} \mathrm{Cd}_{\mathrm{x}} \mathrm{Te}$.

The LMPC HgCdTe APDs which we tested had a quantum efficiency of $>90 \%$ from visible to the of $4.3 \mu \mathrm{m}$ cut-off wavelength. ${ }^{3}$ The differences between the LMPC HgCdTe APDs and the earlier devices are the lower dark noise and even higher APD gain. The preamplifiers are built inside the silicon substrate that is mounted directly underneath the $\mathrm{HgCdTe}$ APD to minimize the stray capacitance. A light barrier is also included between the preamplifier and the HgCdTe APD to minimize the detection of photons emitted by the preamplifier while in operation. ${ }^{1,3}$ Figure 1 shows a schematic of a LMPC $\mathrm{HgCdTe}$ APD pixel. The APD gain is also sufficiently high that the amplitude of the photocurrent pulse from each absorbed photon is many times the noise of the electronics and easily detectable. A unique advantage of HgCdTe APDs is the low excess noise $(\mathrm{F} \approx 1.2)$ that the APD gain for each primary photoelectron is almost deterministic. The amplitude of the output photoelectron pulse is also linear with photon number in the received light pulse.

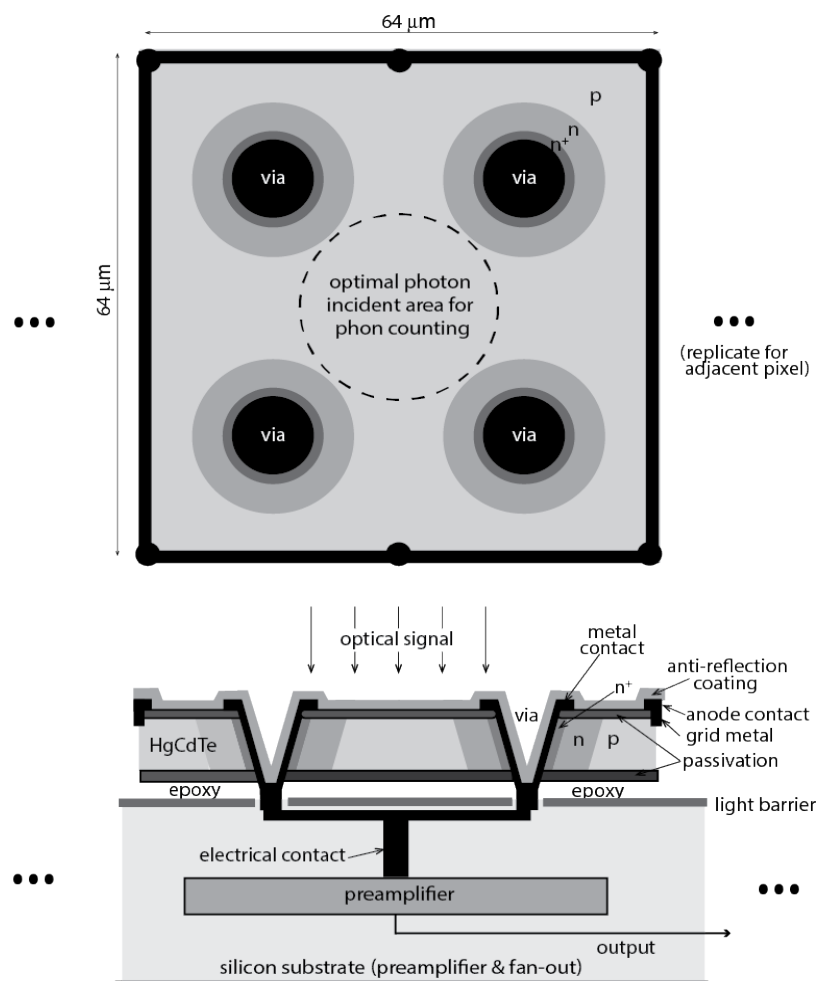

Figure 1. Schematic of a LMPC pixel of HgCdTe APDs by DRS using the HDVIP ${ }^{\circledR}$ p-around-n cylindrical device structure. The APD gain regions are the $n$ - regions around the via. The detectors are vertically integrated to preamplifiers in the underlying silicon readout integrated circuit to reduce the stray capacitance. 
The $\mathrm{n}$ - region around the via is the APD gain region. The incident photoelectrons generated in the p-region that diffuse to the gain region achieve full gain since they traverse the entire length of the gain region. Photoelectrons generated inside the $\mathrm{n}$ - gain junction around the via do not experience the full APD gain and may not be counted. Photons that fall inside the via do not produce photoelectrons. For near zero crosstalk and $100 \%$ fill factor, the optimal region for incident photons is the circular area shown in Fig. 1 in between the four diodes. For the $64 \mu \mathrm{m}$ pixel at high APD gain, the center optimal diameter was $22 \mu \mathrm{m}$ for these $2 \times 8$ FPAs. Areas around and in between the $\mathrm{n}$ - gain regions are also sensitive to single photons, though irregular in shape. The diffusion times for each photoelectron to travel to the APD gain region depend on where photons are absorbed. There is a random time delay, or timing jitter, in the output photoelectron pulses due to the spread of the diffusion times.

The prototype devices we tested had a $2 \times 8$ pixel format at $64 \mu \mathrm{m}$ pitch. There was also a guard ring around the array which consisted of a single row or column pixels biased at the same voltage but not connected to output terminals. The purpose of the guard ring was to have each pixel sees the same boundary conditions to give a more uniform response. Larger array with more pixels can be readily fabricated but limited by the number of output channels that can be accommodated by the chip carrier and the number of feed-through connections in the cryo-cooler housing.

\subsection{Read-out integrated circuits (ROIC)}

The preamplifiers in the read-out integrated circuit (ROIC) are located directly under the HgCdTe APDs. There are 16 channels, one for each pixel, and each consists of a transimpedance amplifier and a unity gain buffer amplifier. The transimpedance gain can be adjusted from 100 to $500 \mathrm{kV} / \mathrm{A}$ but nominally set to $300 \mathrm{kV} / \mathrm{A}$ for photon counting operation. The electrical bandwidth of the ROIC is about $71 \mathrm{MHz} .{ }^{1}$ The combined pulse width of the output is about $6 \mathrm{~ns}$ full width at half maximum (FWHM). ${ }^{1}$ The pulse rise times is about $3 \mathrm{~ns}$ and the fall time is 6-8 ns. The electrical bandwidth of the preamp is mainly limited by the parasitic capacitance at the input terminals according to a full-chip parasitic extraction study using the Hipex software. The single photon input pulse from the detector itself is expected to be a few hundred ps wide as it depends on the transit times of electrons and holes across the multiplication region. We expect the preamplifier bandwidth to be improved significantly through the next few design iterations. The equivalent input noise current spectral density of the transimpedance is about $1.5 \mathrm{~A} / \mathrm{Hz}^{1 / 2}$, which become negligible when the APD gain is $>500$. More detailed description of the LMPC HgCdTe APD array and the ROIC can be found in Beck et al., (2014) and Sullivan et al. (2015).

\subsection{Cryo-coolers}

Liquid nitrogen Dewars were used in the laboratory to characterize the devices for easy exchange of devices and reconfiguration of cold filter and coupling optics and to reduce cost. A liquid nitrogen Dewar was also used as the detector housing to verify APD function and performance at intermediate steps during the microlens installation and the integration of the APD with a closed cycle, permanently sealed, detector-cooler assembly.

A mini-Stirling cooler can be used with these type of HgCdTe APD arrays. These cryo-cooler are originally developed for military use, which are sufficiently rugged to survive a rocket launch and they have demonstrated multi-year lifetimes. ${ }^{10,11}$ We also studied the use a pulse tube cryo-coolers, such as the Micro-cooler from Northrop Grumman. ${ }^{12}$ Both cryo-coolers are suitable for use with these HgCdTe APDs. A pulse tube cooler has a higher efficiency, lower vibration, longer lifetime, but a higher cost.

An integrated detector-cooler assembly (IDCA) was developed under the NASA In-space Validation of Earth Science Technology (InVEST) program. ${ }^{4}$ It consisted of a $2 \times 8$ pixel LMPC HgCdTe arrays in a $1 / 5-\mathrm{W}$ cooler from DRS, ${ }^{11}$ as shown in Fig. 2. The total mass of the cooler was about $700 \mathrm{~g}$ and the electrical power was 4-7 W, depending on the heat sink temperature. The signal buffer amplifiers and the control electronics were mounted around the expander.

\subsection{Microlens arrays}

Microlens arrays may be used to concentrate the incident light onto the center regions of the pixels and to improve the fill factor to nearly $100 \%$ and they were included in the IDCAs developed for the NASA InVEST program . The penalty is they reduce the angle of incidence for the input signals ${ }^{14}$. The microlens arrays were designed and fabricated by JENOPTIC with exactly the same $2 \times 8$ pixel pattern. They were mounted on top of the $2 \times 8$ pixel HgCdTe APD array with a small gap in between. The detector active area is at the focal distance of the microlens array. In use the incident light should be focused at the top of the microlenses. The resulting numerical aperture was $f / 7$. In future applications, the microlens array can be placed closer to the detector to increase the light acceptance angle. 


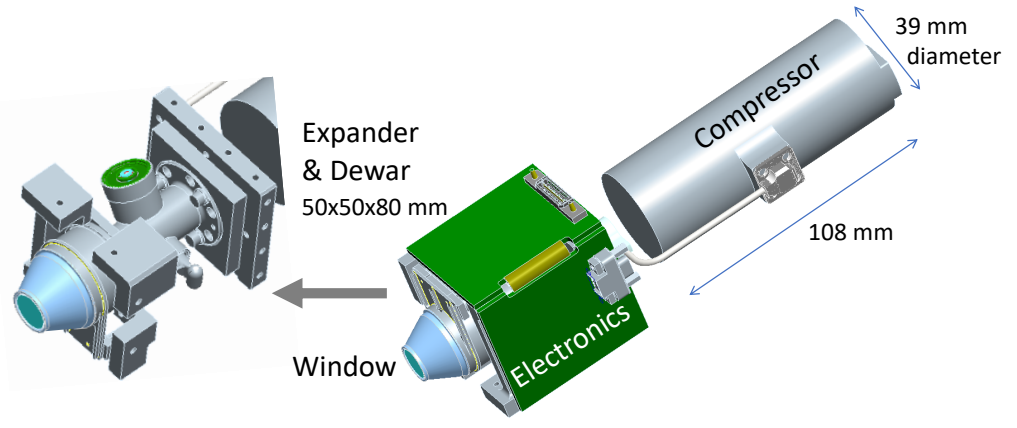

Figure 2. The mini-Stirling cryo-cooler for the 2x8 pixel LMPC HgCdTe APD FPA by DRS.

\section{TEST RESULTS OF THE HGCDTE APD DETECTOR AT GSFC}

The LMPC HgCdTe APD FPAs have been tested extensively tested at DRS prior delivering to NASA GSFC. Those test results have been published in Beck et al. (2014) and Sullivan et al. (2015). Here we report mostly the test results obtained at NASA GSFC using a different test setup. We will first describe the test results of the HgCdTe APD FPA in a liquid nitrogen Dewar. This allowed the test configuration to be easily modified to allow a more thorough device characterization. All the test results were obtained from the analog outputs of the devices. The digital outputs from the internal comparators were verified but the data were not used. We will then describe the test results of the device in a packaged IDCA which were mostly environmental tests.

\subsection{Test setup}

The test setup for detector characterization at GSFC is shown in Fig. 3. There were two test lasers, a generic continuous wave (cw) laser and a pulse laser of pulse width $<100 \mathrm{ps}$ (PicoQuant LDH series), both at $1550 \mathrm{~nm}$ wavelength. A chopper was also used with the cw laser for the responsivity measurements. The light spot on the detector was about $5 \mu \mathrm{m}$ in diameter. The focusing optics assembly was mounted on a X-Y translation stage which was controlled by a computer to scan the light spot across the detector at $1-\mu \mathrm{m}$ step size. An optical fiber attenuator was used to adjust the signal power. There was also a fixed attenuator $(30 \mathrm{~dB})$ in the collimated space of the focusing optics assembly. Both of them were calibrated before the measurements. The optical signal power onto the detector at $0 \mathrm{~dB}$ attenuation was calibrated by replacing the detector with the optical power meter with the optical fiber attenuator set to $0 \mathrm{~dB}$ and the fixed attenuator removed. There was a 16-to-1 switch to select the pixel output to be measured and a linear amplifier with a voltage gain of 5 to further amplify the signal to above the noise floor of the test equipment.

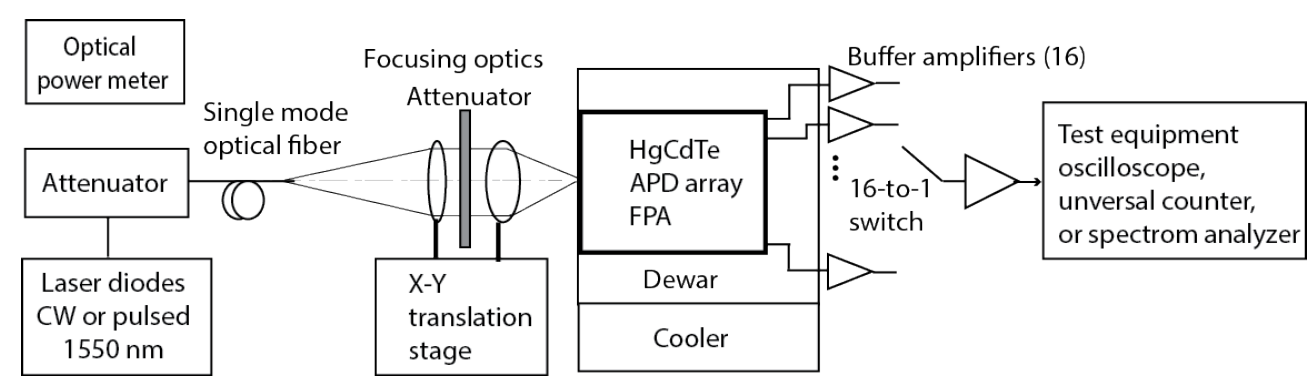

Figure 3. Block diagram of the test setup for detector characterization at GSFC. The cooler was either a liquid nitrogen Dewar during the initial characterization or a mini-Stirling cryo-cooler for the IDCA testing.

\subsection{Responsivity and APD gain}

The detector responsivity in (V/W) from a single pixel was measured as a function of the APD bias voltage using the cw laser. A mechanical chopper was used in between the focusing lenses. The signal being measured was the difference of the buffer amplifier output with light blocked and unblocked by the chopper. The use of the chopper helped to subtract the baseline from a relatively weak signal. The actual APD bias was the sum of the external bias voltage and the $0.95 \mathrm{~V}$ DC 
offset at the input of the preamplifiers. Since the photoelectron multiplication gain of the HgCdTe APDs is unity at a net bias of $<1.0 \mathrm{~V}, 2,7$ the APD gain at other bias voltages could be obtained by dividing the responsivity at a given bias voltage by that at zero external bias voltage. Figure 4 plots both the responsivity and the APD gain of a typical pixel as a function of the APD bias voltage (not including the $0.95 \mathrm{~V}$ from the preamplifier, same for the rest of the paper). The output became saturated at APD bias voltage above $9 \mathrm{~V}$, possibly due to preamplifier saturation under this relatively high and near $\mathrm{cw}$ illumination (10 pW/pixel). The APD gain should increase exponentially with the bias voltage, as shown by the dotted line and crosses in Fig. 4. The responsivity shown in Fig. 4 includes the gain of the built-in buffer amplifier but not the amplifier before the test equipment. The HgCdTe APD quantum efficiency was estimated to be about $90 \%$ assuming unity APD gain at $0 \mathrm{~V}$ biase, preamplifier gain of $200 \mathrm{kV} / \mathrm{W}$, buffer amplifier gain of $5 \mathrm{x}$, and $1 \mathrm{~dB}$ cable loss.

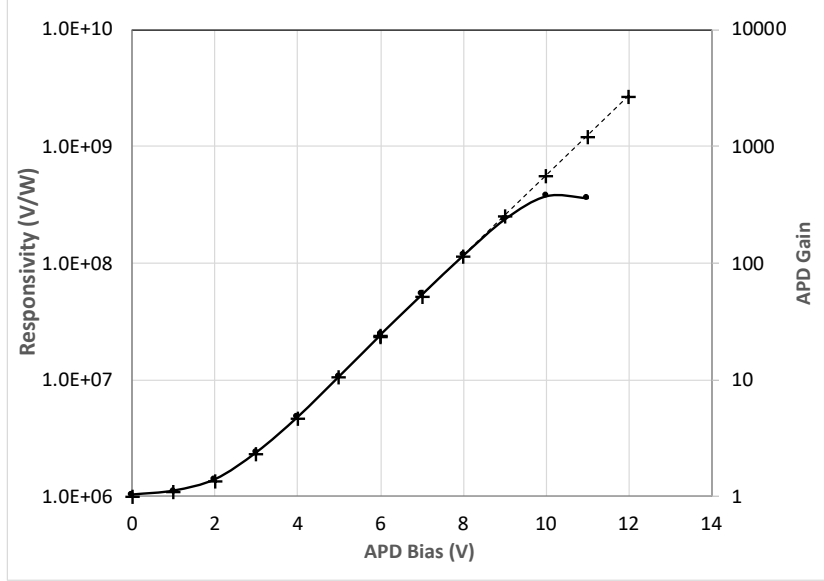

Figure 4. Measurement data of the device responsivity in V/W and the APD gain as a function of the APD bias voltage from one of the pixel (R2:C3) of FPA-A8327-8-2 using a $1550 \mathrm{~nm}$ cw laser and a mechanical chopper. The dotted line and crosses in the plot shows the extrapolated APD gain assuming the saturation in the detector output was caused by the preamplifier.

\subsection{Noise spectral density and NEP}

The detector output noise spectra were measured using an RF spectrum analyzer. Figure 5 shows the noise output from a single pixel under a relatively strong $\mathrm{cw}$ laser light illumination and dark noise only condition. The net noise spectral density in response to the illumination is also plotted. The detector electrical bandwidth can be estimated as the frequency where the net illuminated noise density drops by $3 \mathrm{~dB}$, which is about $40 \mathrm{MHz}$ in this case.

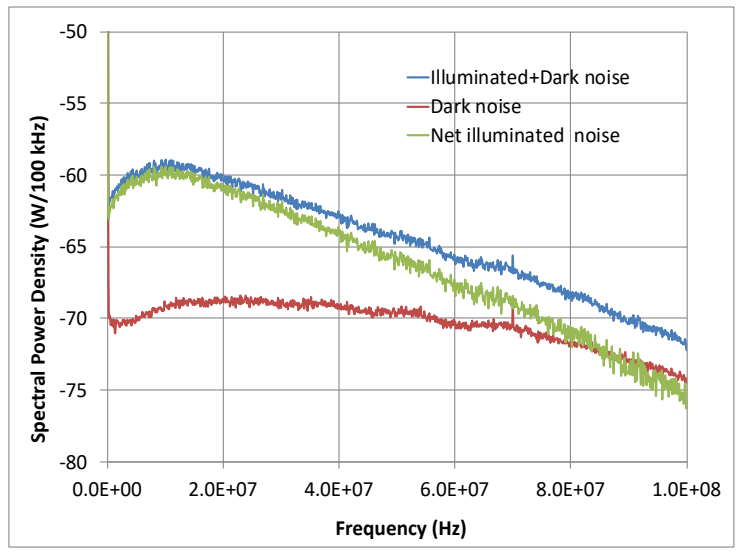

Figure 5. Detector output noise spectral density under a relatively strong illumination and dark conditions, and the difference from Pixel (R2:C3) of FPA-A8327-8-2. The noise spectral densities shown were the actual measurement by the spectrum analyzer, including the gain of the amplifier before the test equipment. The electrical bandwidth was about $40 \mathrm{MHz}$ as estimated from the net illuminated noise. 
The detector noise density was measured as a function of the APD bias voltage. The detector noise equivalent power (NEP) was calculated by converting noise power to rms voltage noise in $\mathrm{V} / \mathrm{Hz}^{1 / 2}$ and then dividing it by the responsivity, as shown in Fig. 6. The NEP became sufficiently low to allow reliable single photon detection when the APD bias was $>11 \mathrm{~V}$.

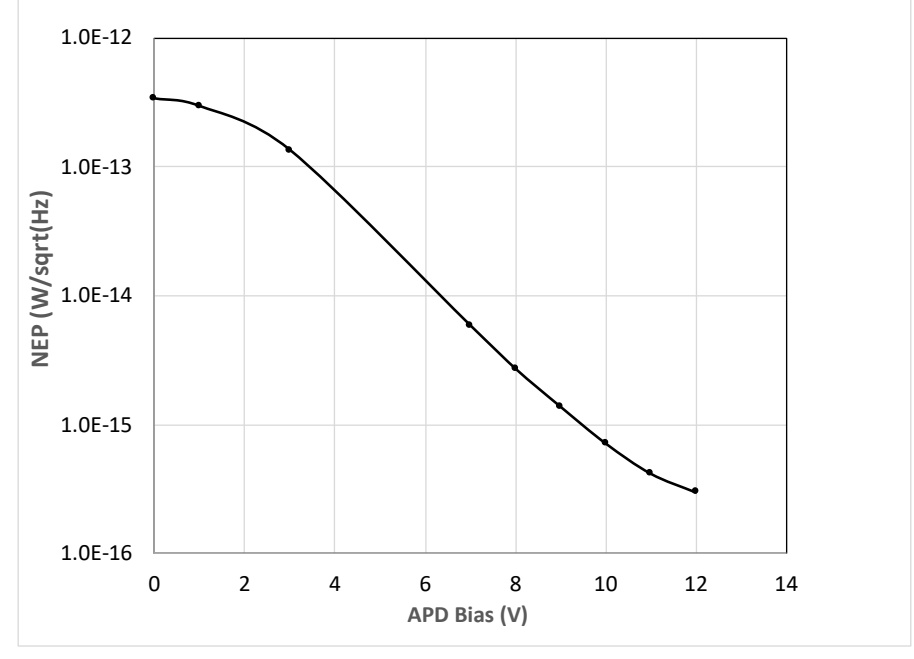

Figure 6. The noise equivalent power (NEP) vs. APD biase voltage from Pixel (R2:C3) of FPA-A8327-8-2. The electrical bandwidth was about $40 \mathrm{~Hz}$ as estimated from the net illuminated noise.

\subsection{Pulse responses, timing jitter, dynamic range, and APD excess noise factor}

The detector analog output pulse waveforms were measured using the short pulse laser and a high-speed oscilloscope. Figures 7 shows the measured pulse waveforms at zero APD bias (gain=1) and 11.5 V APD bias. The laser signal level was about 15 photons/pulse for the latter case. Because the pulse width was narrow $(<100 \mathrm{ps})$, the photons could be assumed to arrive at the same time.
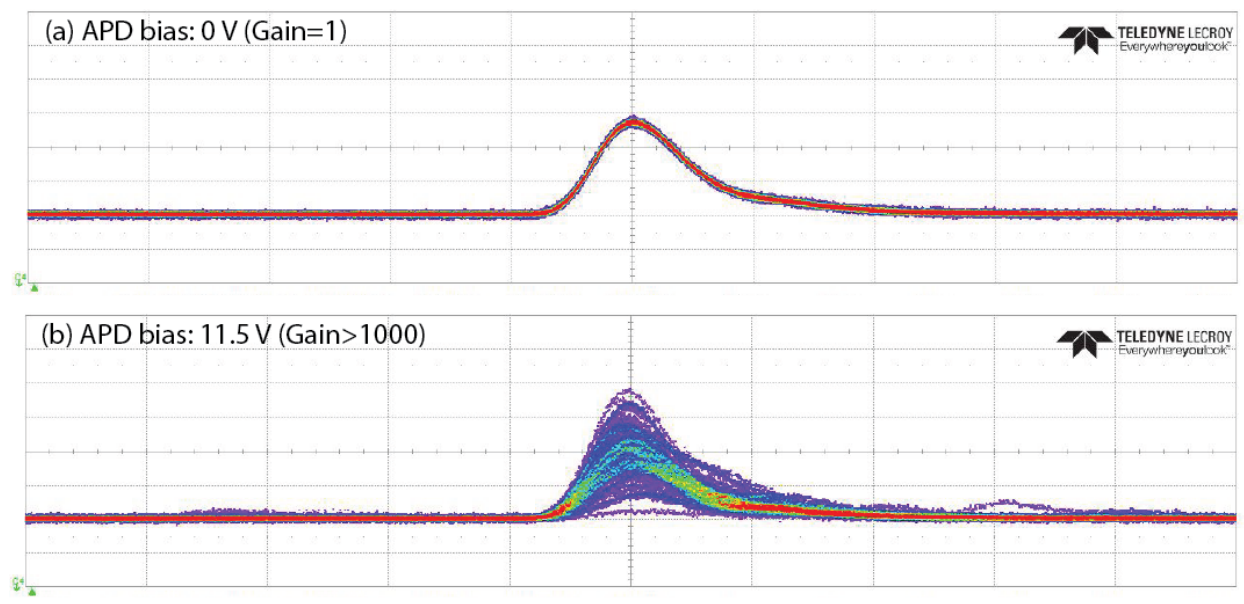

Figure 7. Output pulse waveform of Pixel (R2:C3) of FPA-A8327-8-2 in response to a narrow pulse width $(<100 \mathrm{ps})$ laser illumination at 0 and $11.5 \mathrm{~V}$ APD bias.

The pulse rise and fall times were measured to be $3.2 \mathrm{~ns}$ and $7.4 \mathrm{~ns}$, respectively. The pulse width was about $8 \mathrm{~ns}$ full width at half maximum (FWHM). The oscilloscope was triggered by the timing pulses from the laser source. The oscilloscope also gave the timing jitter of the pulse waveforms at $50 \%$ pulse amplitude. Since the timing pulse from the laser was synchronized with the laser pulses with little timing jitter $(\sim 15 \mathrm{ps})$, the timing jitter reported by the oscilloscope were 
largely the detector timing jitter. The jitter was $80 \mathrm{ps}$ when the APD was biased at $0 \mathrm{~V}$ (top panel) and $290 \mathrm{ps}$ when the APD was biased at $11.5 \mathrm{~V}$ (bottom panel).

The pulse amplitude was measured as a function of the incident laser photons/pulse at $0,7.5$, and $11.5 \mathrm{~V}$ APD bias using the same pulsed laser but over a range of the input optical signal level. The mean and standard deviation of the pulse amplitude of the measurements are plotted in Fig. 8. The linear dynamic range of the detector output at each APD gain setting was about two orders of magnitude. The total detector output dynamic range was about five orders of magnitudes when adjusting the APD bias voltage.

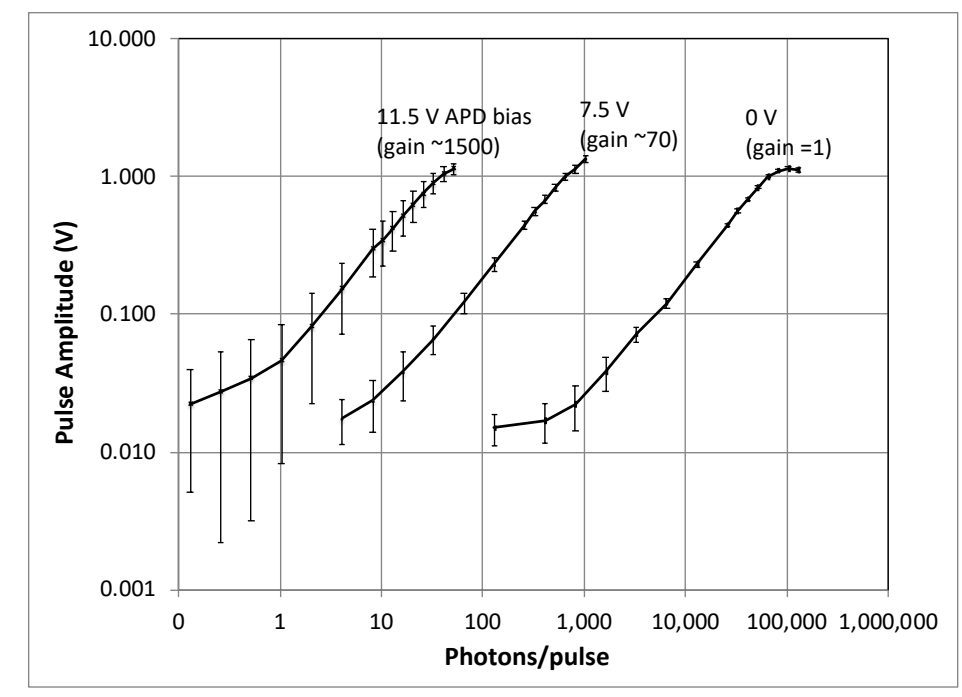

Figure 8. Pulse amplitude, mean and standard deviation (error bars) of the output from Pixel (R2:C3) of FPA-A8327-8-2 under a narrow pulse width $(<100 \mathrm{ps})$ laser illumination at $0,7.5$, and $11.5 \mathrm{~V}$ APD bias.

The APD gain excess noise factor, $F_{e x}$, could be solved approximately from the equation when the dark noise and the electronics noise could be neglected, ${ }^{2}$

$$
\frac{\mu_{\text {sig }}}{\sigma_{\text {sig }}}=\frac{\eta_{Q E}\langle G\rangle\left\langle n_{\text {sig }}\right\rangle}{\sqrt{\eta_{Q E} F_{e x}\langle G\rangle^{2}\left\langle n_{\text {sig }}\right\rangle}}
$$

where $\mu_{\text {sig }}$ and $\sigma_{\text {sig }}$ are the mean and standard deviation of the output pulse amplitude, $\eta_{Q E}$ is the detector quantum efficiency assuming to be $90 \%,\langle G\rangle$ is the average APD gain, and $\left\langle n_{\text {sig }}\right\rangle$ is the average number of incident photons in the laser pulses. The resulting excess noise factor was 1.16 to 1.30 using the data from 10 to 20 photons/pulse at $11.5 \mathrm{~V}$ APD bias voltage.

\subsection{Photon counting performance}

The detector could be used for single photon counting with the use of a discriminator (comparator) and the APD greater than 500. Figure 9 shows the measured dark count rate and the total net count rate vs. the incident photon rate from the cw laser. The discriminator was set to the optimal value where the photon detection efficiency (PDE) was optimal. The APD bias was set to $11.5 \mathrm{~V}$. The results from two pixels were plotted and they were nearly the same. The dark count rate was about 250,000/s. A major portion of the dark counts were believed to come photons emitted by the ROIC and from the thermal photons leaked through the perimeters of the cold shield. The dark current measured from the test APDs on the same wafer showed the net dark count rate should be about 15,000/s. The PDE was about 70\% before the digital output started to saturate due to the finite pulse width and the electrical bandwidth. The statistics of the detected photons closely followed Poisson distributions. The detected photon count rate started to saturate at about 10 million counts/s, that was limited by the electrical bandwidth of the preamplifier. The dynamic range can be extended by using the analog signal output waveform for signal levels above the saturation point of the single photon-counting channel. 


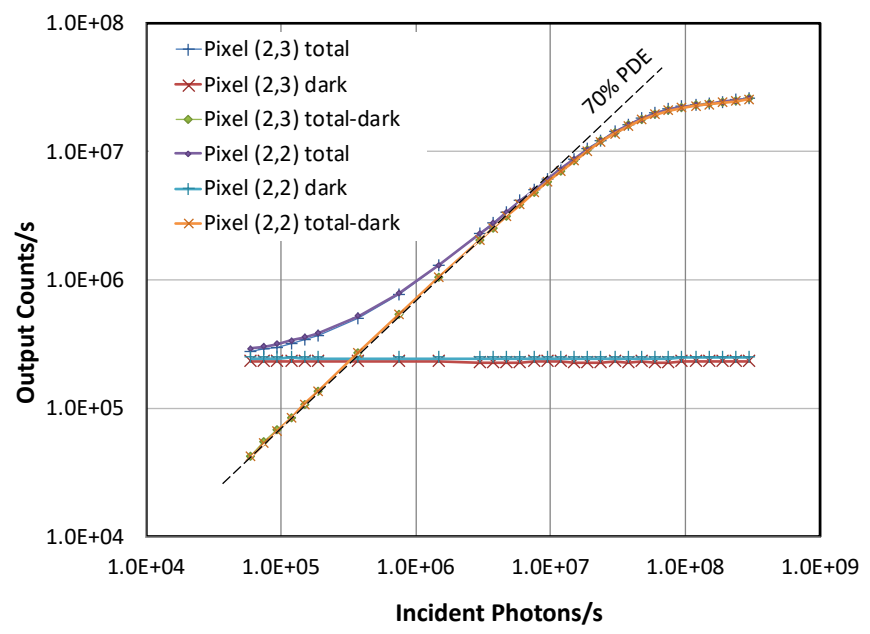

Figure 9. Detected photon rate vs. the incident photon rates from two adjacent pixels of FPA-A8327-8-2 at the optimal discriminator threshold level and an APD bias of $11.5 \mathrm{~V}$.

The PDE vs. false event rate (FER) was measured over a range of discriminator thresholds at different APD bias voltage from another device and the results are plotted in Fig. 10. The PDE and FER increased as the discriminator threshold decreased until the counter saturated. The optimal threshold should be where the PDE reached its maximum but before saturation. The APD bias should be above 10.5V (APD gain >500) for single photon counting.

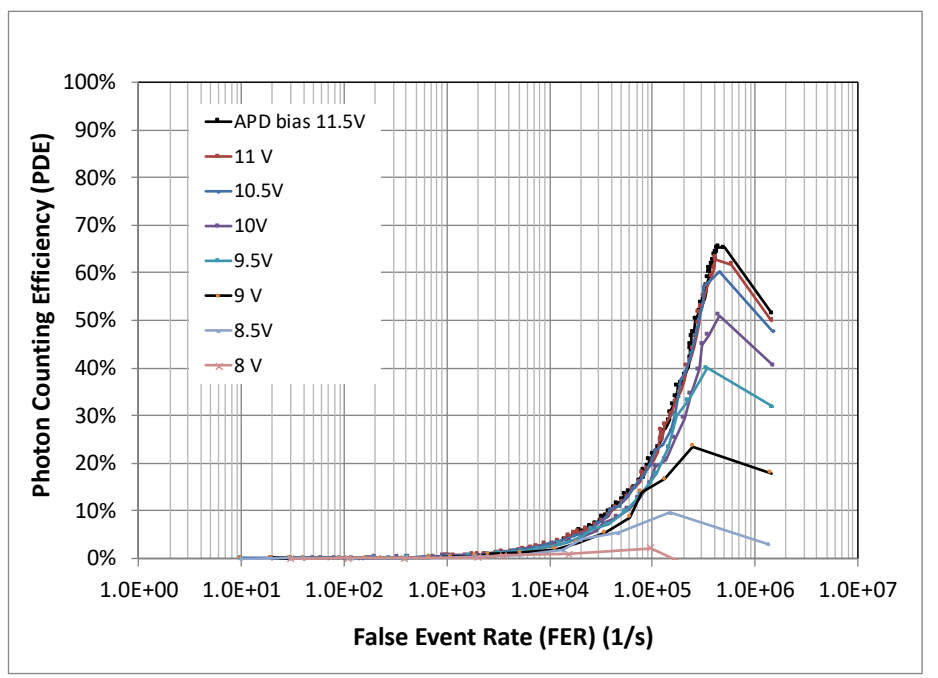

Figure 10. Photon counting efficiency (PDE) vs. false event rate (FER) of FPA-A8327-14-1, Pixel (1,6), over a range of APD bias voltage.

The PDE vs. FER were measured again at different device temperature at a fixed APD bias of $11.5 \mathrm{~V}$. It shows the device temperature could actually be set to $110 \mathrm{~K}$ instead of $80 \mathrm{~K}$ for the photon counting performance, consistent with the results from a similar type of $\mathrm{HgCdTe}$ APD arrays. ${ }^{2}$ 


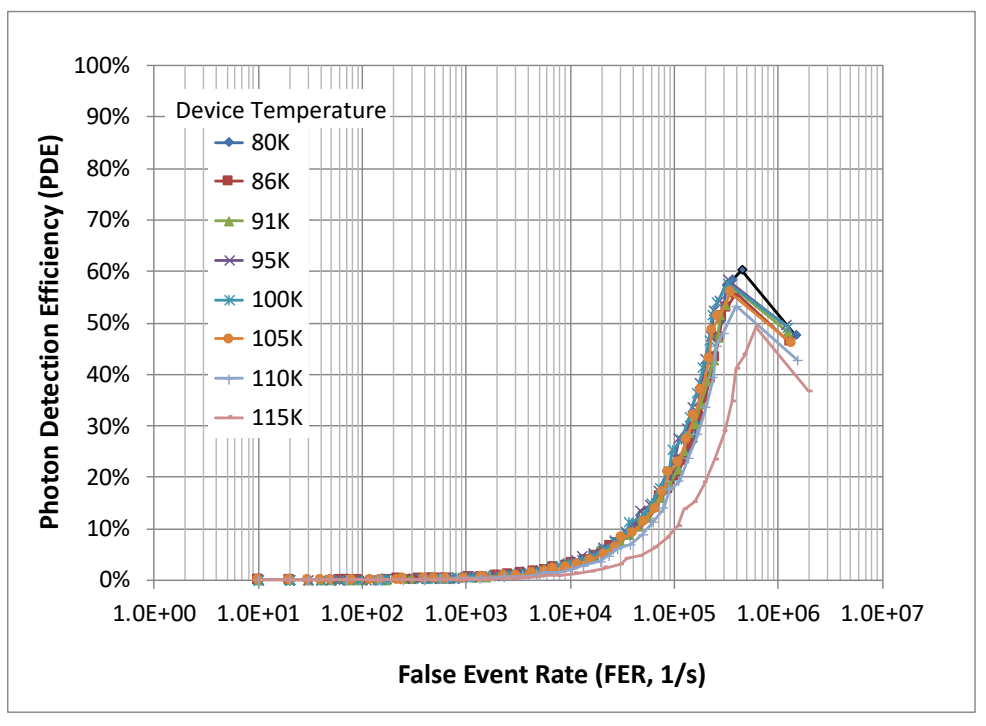

Figure 11. Photon counting efficiency (PDE) vs. false event rate (FER) of FPA-A8327-14-1, Pixel (1,6), over a range of device temperature. The APD bias voltage was set to $11.5 \mathrm{~V}$.

\subsection{Active area}

The active area of the detector was measured by scanning the light spot across a pixel in a raster pattern while recording the photon count rate with the counter. The result is shown in Fig. 12. There were four low signal voids, one for each of the four diodes in the pixel. The center region has a diameter of about $22 \mu \mathrm{m}$. A microlens array may be used to concentrate the light on the center spot to achieve near 100\% fill factor at the cost of reduced light acceptance angle.

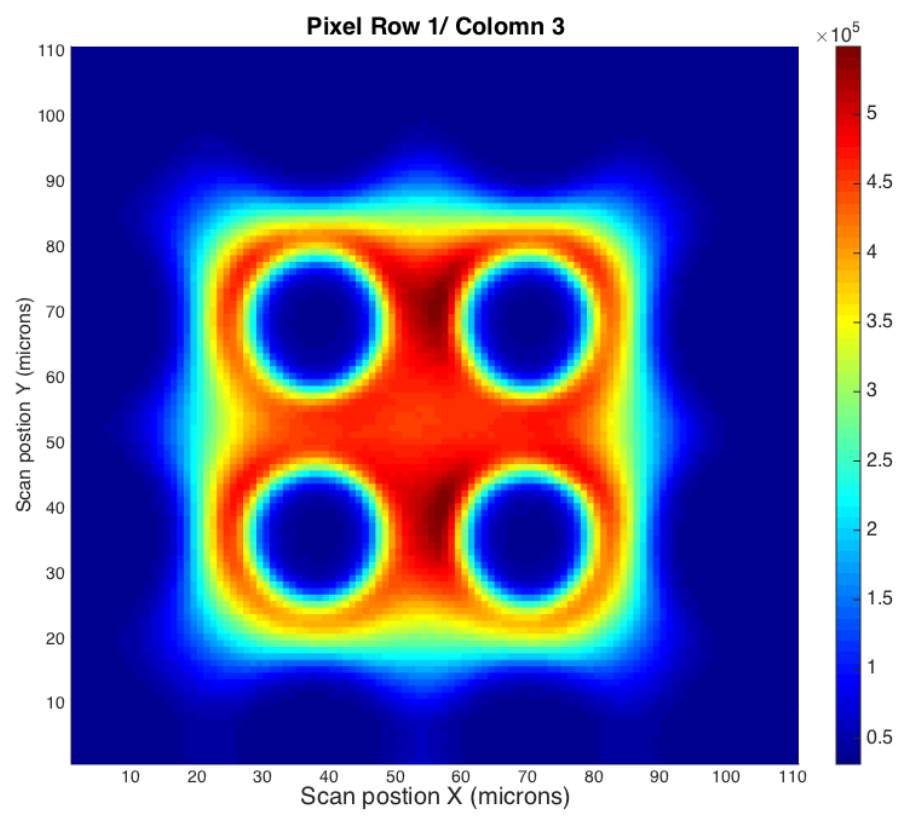

Figure 12. Detector active area surface map obtained via a raster scan of the laser spot while measuring the photon counts of the of FPA-A8327-14-1, Pixel (1,3). The APD bias voltage was set to $11.5 \mathrm{~V}$. 
The detector active area in analog mode was also measured by recording the output pulse amplitude while raster scanning the laser spot at APD bias of $0 \mathrm{~V} .10 .5 \mathrm{~V}$, and $11.5 \mathrm{~V}$. Figure 13 shows the normalized surface map. The diameter of the signal voids increased with the APD bias due to increasing gain in the $\mathrm{n}$ - region around the via. Photons falling in the gain region were not multiplied by the full APD gain resulting in the gain region becoming optically inactive relative to signals generated outside the gain region that achieve full gain. These results are consistent with that predicted by the model. ${ }^{14}$
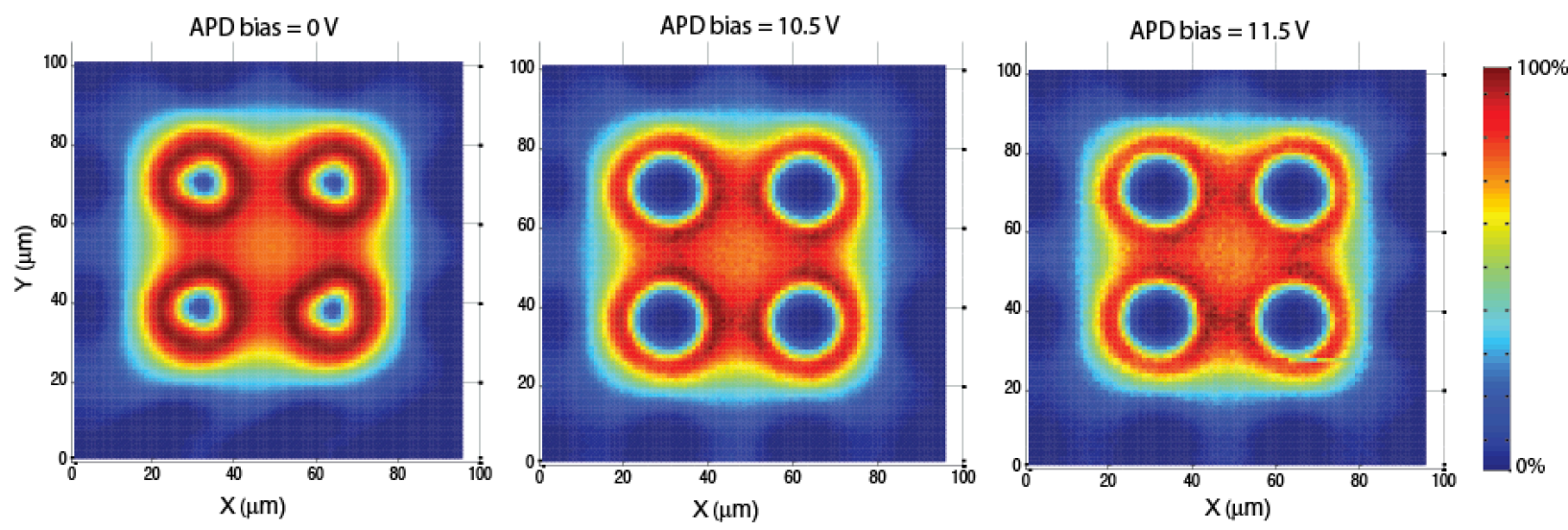

Figure 13. Detector active area surface map of FPA-A8327-8-2, Pixel (2,3), at APD bias voltage of 0 V, $10.5 \mathrm{~V}$ and $11.5 \mathrm{~V}$ obtained by measuring the detector output pulse amplitude and raster scanning the laser spot position across the pixel.

\section{RESULTS FROM THE INTEGRATED DETECTOR COOLER ASSEMBLY TESTING}

\subsection{IDCA developed for a CubeSat}

There were two IDCAs delivered by DRS and tested at GSFC. Figure 14 shows a photograph of the as delivered IDCA. A mu-metal magnetic shield was used around the compressor for the CubeSat application. An L-shaped aluminum bracket was used as the base plate as well as the heat sink. The mu-metal weight was $350 \mathrm{~g}$, and the bracket weight $250 \mathrm{~g}$. The cooler control module, the electronics around the expander and harnesses weigh about $100 \mathrm{~g}$. The total mass of the IDCA including the $700 \mathrm{~g}$ cryo-cooler was $1.4 \mathrm{~kg}$. The cooler was capable of cooling the detector to either $80 \mathrm{~K}$ or $110 \mathrm{~K}$. The environmental tests were performed with the detector temperature set to $110 \mathrm{~K}$, since it had been shown to give the same performance, and significantly reduced the electrical power consumption and extended the cooler lifetime. ${ }^{2}$ Microlens arrays were included in both IDCA units. There were two cold filters in cascade in the Dewar with a pass band from 0.9 to $2.1 \mu \mathrm{m}$. The cold shield had a numerical aperture of $\mathrm{f} / 7$, which matched the light acceptance angle of the detector with the microlens array.

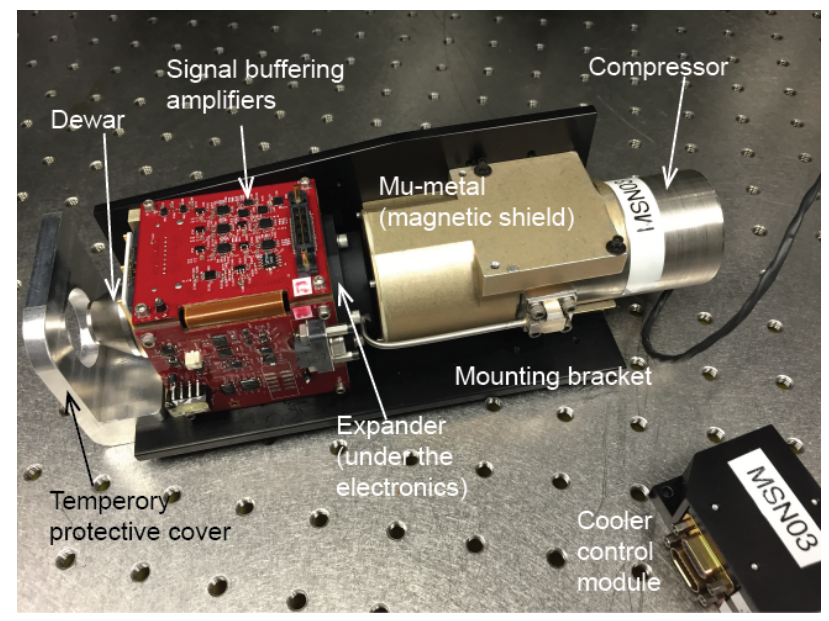

Figure 14. Photograph of the IDCA developed for a CubeSat program. 
A series of environmental tests were performed to the IDCAs to demonstrate their technology readiness level (TRL) for a CubeSat space mission. ${ }^{4}$ These include vibration tests to verify the mechanical integrity of the unit; thermal tests to verify that the cooler and the electronics could operate over the entire temperature range predicted for the CubeSat mission; and a thermal vacuum test to verify the IDCA can operate in a space-like environment. The rest of this section described the test results. A series of radiation damage tests of the $\mathrm{HgCdTe}$ APD arrays were also performed and the results will be published separately.

\subsection{Vibration tests}

Vibration tests were performed on the IDCA per standard test procedure for the DRS mini-Stirling cooler for military applications. The first vibration test was to the cooler assembly up to $14 \mathrm{G} \mathrm{rms}$ with the detector, microlens array, and cold shield installed but before the Dewar was capped and sealed. The second test was performed to the entire IDCA prior to delivery up to $10 \mathrm{G}$ rms. When operating, the vibration levels of the cryo-cooler assembly were measured to be about 0.05 $\mathrm{g}$ root-mean-squared (rms) in all three axes, with most of the energy at $60 \mathrm{~Hz}$ along the Dewar optical axis due to the motion of the piston in the expander. The frequencies of other significant harmonics are all above $1 \mathrm{kHz}$ in all three directions.

\subsection{Thermal tests}

A thermal shock test was performed to the IDCA at DRS by placing the IDCA in a temperature chamber for four hours and moved to $71^{\circ} \mathrm{C}$ within one minute. A thermal cycle test was also performed at DRS from $-34^{\circ} \mathrm{C}$ to $71^{\circ} \mathrm{C}$ under forced air at $-34^{\circ} \mathrm{C}$ with the unit powered on at $-24^{\circ} \mathrm{C}$ and $60^{\circ} \mathrm{C}$ with the detector temperature set to $80 \mathrm{~K}$.

A more extended thermal test was performed at NASA GSFC over the predicted CubeSat temperatures plus margin. The IDCA was tested for five more cycles from $-20^{\circ} \mathrm{C}$ to $60^{\circ} \mathrm{C}$, which, plus the one temperature cycle at DRS, satisfied the Goddard Environmental Verification Standard (GEVS) for thermal tests. The ICDA was under forced air and all the subsystems were at the same temperature. The detector temperature was set to $80 \mathrm{~K}$ and $110 \mathrm{~K}$. The detector dark count rate and the total count rate from all pixels under a fixed illumination were monitored and they were consistent throughout the tests. The electrical power of the cooler and the electronics were monitored and the results are plotted in Fig. 15. It shows the total power at room ambient temperature was about $6 \mathrm{~W}$ with the detector set to $110 \mathrm{~K}$, which is comparable or less than a power for a multi-stage thermal electrical cooler.

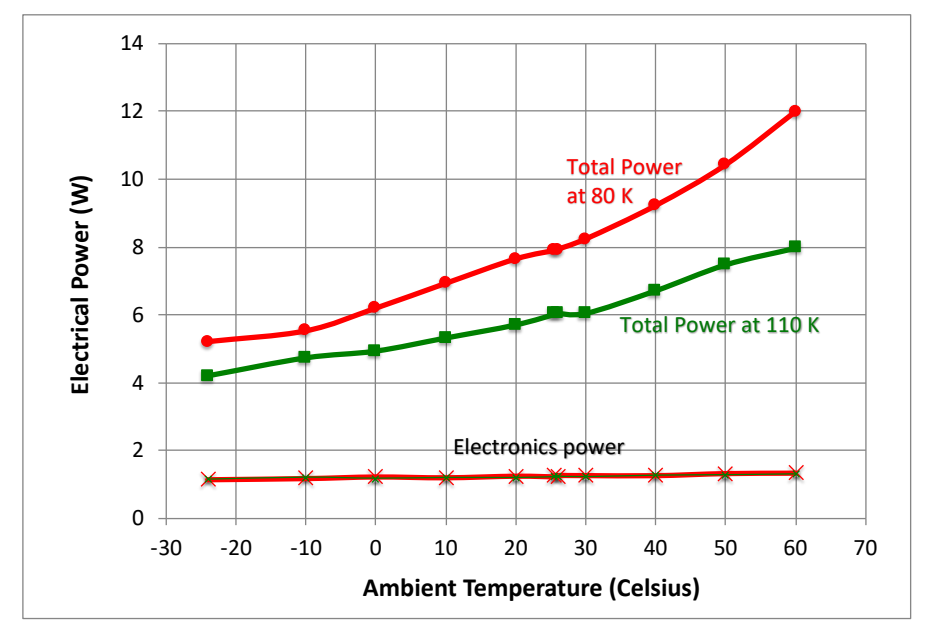

Figure 15. Electrical power for the cryo-cooler and the electronics (buffer amplifiers and the digital controls).

\subsection{Microlens array performance}

The microlens array stayed attached and aligned throughout the vibration and thermal tests. There was no sign of any change in the optical throughput based on the total photon count rates under the fixed illumination.

A raster scan of the detector active area with the microlens array was performed and the result is plotted in Fig. 16. The light spot size in this scan was larger due to vignetting of the laser light by the F/7 cold shield and the result was not as 
sharp as those shown in Fig. 12. Nevertheless, it showed the microlens array functioned as expected and improved the fill factor to $100 \%$.

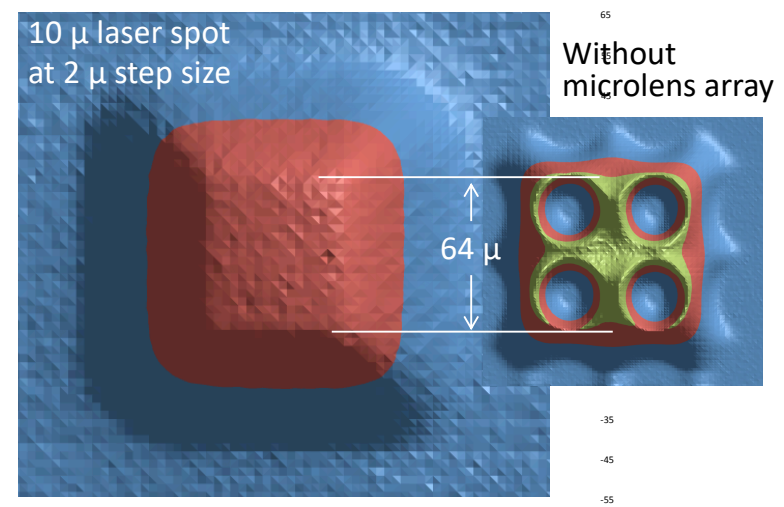

Figure 16. IDCA pixel active area scan with the microlens array compared to earlier scan result without microlens array.

\subsection{Thermal vacuum test}

A thermal vacuum test was performed on one of the IDCA units. Four cycles were performed between $-20^{\circ} \mathrm{C}$ and $40^{\circ} \mathrm{C}$. The detector temperature was set to $110 \mathrm{~K}$ and regulated to $\pm 0.1^{\circ} \mathrm{C}$. The unit was covered with thermal blanket to minimize the radiative heat transfer. The main thermal path was through the mounting bracket. The temperatures of the expander and the compressor were nearly the same. The electronics circuit board and the cooler control module were $5-10^{\circ} \mathrm{C}$ warmer. The test lasted for about 2 weeks. The electrical power from the power supplies were monitored throughout the test and the results were plotted in Fig. 17. The total electrical power for the entire IDCA was 5-7 W with the detector temperature at $110 \mathrm{~K}$.

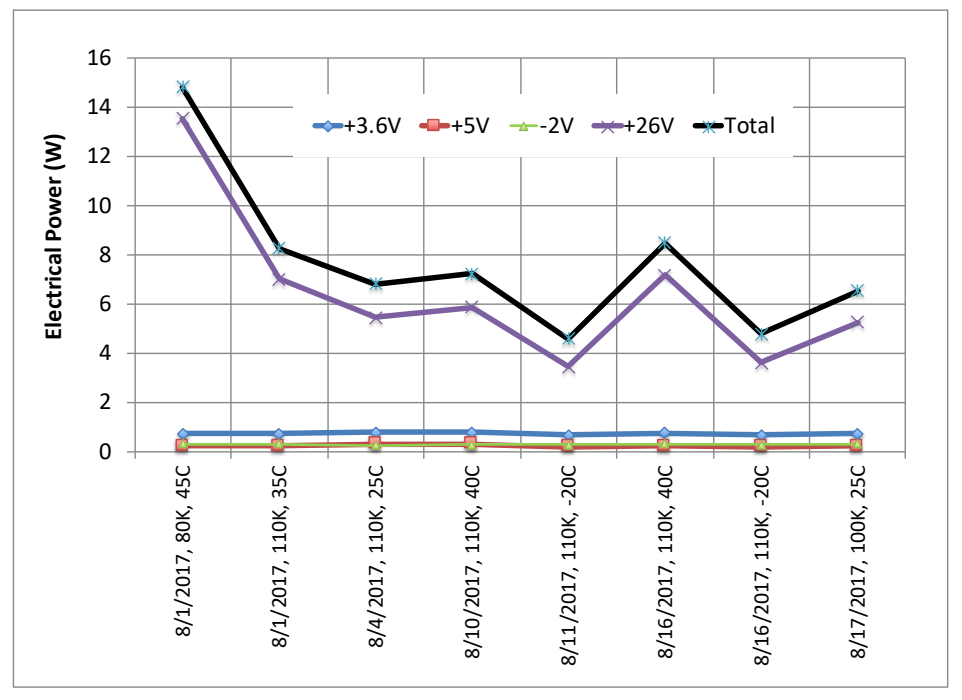

Figure 17. Electrical power from each and all power supplies to the IDCA during the thermal vacuum test.

The detector dark count rate and total count rate were monitored throughout the tests under a constant illumination. They were consistent and repeatable at a given temperature. One issue identified was that the cold shield inside the Dewar had light leaks around the base area that the "dark" count rates varied with the Dewar body temperature to 10-100 times the intrinsic detector dark count rate. There were also spurious leakages in the cold filter beyond the passband but still with the detector spectral response in the mid infrared wavelength region. We believe that the cold shield can be modified to significantly reduce the leakage. A new cold filter from a different supplier has already been used in later devices that has sufficient out-of-band attenuation. 


\section{SUMMARY}

A set of linear mode photon counting (LMPC) $\mathrm{HgCdTe}$ avalanche photodiode (APD) detectors were developed by Leonardo DRS for space lidar applications at NASA. The devices had 2x8 pixels at $64 \mu \mathrm{m}$ spacing. The LMPC HgCdTe APDs have demonstrated $>60 \%$ photon detection efficiency from 0.9 to $4.3 \mu \mathrm{m}$ wavelengths and had about $250,000 / \mathrm{s}$ dark count rate. The devices gave linear analog waveform output with a wide dynamic range. An integrated detector cooler assembly (IDCA) has been developed with the 2x8 pixel LMPD HgCdTe APD array and a ruggedized mini Stirling cryocooler. A microlens array has been integrated with the APD array that has shown to improve the detector fill factor to $100 \%$. The IDCA has passed vibration, thermal cycle, and thermal vacuum tests per NASA Standard (GEVS). A series of radiation tests were also performed which showed the devices can be used in a typical multi-year Earth or planetary science mission. ${ }^{15}$

\section{ACKNOWLEDGEMENTS}

We thank NASA's Earth Science Technology Office (ESTO) for its continued support for developing this space lidar detector. We thank Guangning Yang of NASA GSFC and Shawn Perdue of Aerospace Corp. for their assistance in the testing at GSFC.

\section{REFERENCES}

[1] Beck, J. D., Scritchfield, R., Mitra, P., Sullivan III, W. W., Gleckler, A. D., Strittmatter, R., and Martin, R. J., "Linear mode photon counting with the noiseless gain HgCdTe e-avalanche photodiode," Opt. Eng. 53(8), 081905 (2014).

[2] Sun, X, Abshire, J. B., Beck, J. D., Mitra, P., Reiff, K., and Yang, G., "HgCdTe avalanche photodiode detectors for airborne and spaceborne lidar at infrared wavelengths," Optics Express, 25(14) 16589-16602 (2017).

[3] Sullivan, W., Beck, J., Scritchfield, R., Skokan, M., MItra, P., Sun, X., Abshire, J. Carpenter, D., and Lane, B., "Linear-mode HgCdTe avalanche photodiodes for photon-counting applications," J. Electron. Mater. 44(9) 3092$3101(2015)$.

[4] Fields, R, Sun, X., Abshire, J., Beck, J., Rawlings, R. M., and Hinkley, D., “A linear mode photon-counting (LMPC) detector array in a CubeSat to enable earth science LIDAR measurements," Proceeding of the International Geoscience and Remote Sensing Symposium (IGARSS), 5312-5315, Paper FR2.B1 (2015).

[5] Sun, X., Abshire, J. B., Lauenstein, J. M., Sullivan III, W., Beck, J., and Hubbs, J. E., "Evaluation of space radiation effects on HgCdTe avalanche photodiode arrays for lidar applications," Infrared Technology and Applications XLIV, SPIE 10624, Paper No. 10624-15 (this conference) (2018).

[6] Beck, J., Wan, C., Kinch, M., Robinson, J., Mitra, P., Scritchfield, R., Ma, F., and Campbell, J., "The HgCdTe electron avalanche photodiode," J. Electron. Mater. 35(6), 1166-1173 (2006).

[7] Kinch, M. A., [Fundamental of Infrared Detector Materials], SPIE Press, Bellingham, ch. 7 (2007).

[8] Baker, I. and Kinch, M, "HgCdTe electron avalanche photodiodes (EAPDs), in Mercury Cadmium Telluride: Growth, Properties and Applications, Capper, P. and Garland, J. ed. (John Wiley \& Sons, Chichester, ch. 21 (2011).

[9] Kinch, M. A., [State-of-the-Art Infrared Detector Technology], SPIE Press, Bellingham, ch. 6 (2014).

[10] Veprik, A, Zehtzer, S., Pundak, N., and Riabzev, S., Compact linear split Stirling cryogenic cooler for high temperature infrared imagers, Cryocoolers. 16, 121-132 (2011).

[11] Rawlings, D, and G. Averitt, A linear Drive Cryocooler for Ultra-Small Infrared Sensor Systems, Proc. SPIE 9070, P90702R (2014).

[12] Raab, J., and Tward, E., Northrop Grumman Aerospace Systems cryocooler overview, Cryogenics 50, $572-581$ (2010).

[13] Southwell, H. W., Focal-plane pixel-energy redistribution and concentration by use of microlens arrays, Appl. Opt. 33 (16), 3460-3464 (1994).

[14]Beck, J., Kinch, M., and Sun, X., Update on linear mode photon counting with the HgCdTe e-APD, Optical Engineering, 53 (8), 081906 (2014).

[15] Sun, X., Abshire, J. B., Lauenstein, J. M., Sullivan III, W., Beck, J., and Hubbs, J. E., Evaluation of space radiation effects on $\mathrm{HgCdTe}$ avalanche photodiode arrays for lidar applications, to be presented at Proc. SPIE 10624 10624-15 (2018). 